\title{
Modeling, Identification, and Simulation of Positional Displacement Control for Ribbon Bridges
}

\author{
Van Trong Nguyen ${ }^{1}$, Yong-Woon $\mathrm{Choi}^{2}$, Jung-In Yoon $^{3}$, Kwang-Hwan Choi ${ }^{3}$, Chang-Hyo \\ $\mathrm{Son}^{3}$, and Young-Bok Kim ${ }^{1, *}$ \\ ${ }^{1}$ Department of Mechanical System Engineering, Pukyong National University, Busan, Republic of \\ Korea \\ ${ }^{2}$ Department of Information System Science, Soka University, Tokyo, Japan \\ ${ }^{3}$ Department of Refrigeration and Air-conditioning Engineering, Pukyong National University, Busan, \\ Republic of Korea
}

\begin{abstract}
The demand of river-crossing is steadily increasing, the speedy and safety are two most important factors that decide the success of the applied solutions. This paper partially proposes a method for ribbon bridge installation and self-correction by describing the planar motion for a ribbon bridge, including several experimentally identified over a certain range of speeds. The mathematical modeling and system identification aim to provide a model that is sufficient for allowance of model-based control designs. In order to design the stable yaw control system of auto-correction of positional displacement, the Linear-Quadratic Regulator (LQR) was employed for yaw controller. Accordingly, the numerical study was carried out under a variety of disturbances to verify the system modeling and the efficiency of the designed controller. The simulation results demonstrate that the proposed controller has promising feasibility of the yaw placement automatic correct during operation and further extended results of the whole bridge system.
\end{abstract}

\section{Introduction}

The river-crossing operation is one of the most necessary operations for emergency restoration in military and other viewpoints. The major objective of river-crossing is transporting the armed forces between two shores of the river, lake, or a canal. In term of bridging, the improved ribbon bridge is one of the most effective solutions because of its transportation ability and the portability. It is constructed on a river by jointing multiple floating bays [1] to connect two river sides. Conventionally, the linearity of Ribbon Bridge is maintained by employing the assistances of a number of erection boats. Therefore, the river-crossing contains a certain risky factors in military standpoint, especially during the combat situation. The safety and speedy are two crucial factors that decide the success of river-crossing process. In reality, a number of operators are required to install and to retain

\footnotetext{
*Corresponding author: kpjiwoo@pknu.ac.kr
} 
the linearity of the ribbon bridge by adjusting each floating bay. Additionally, it is strongly difficult to maintain the placement of a single floating unit by the erection boats due to many other affections including water flow current, moving load, especially under harsh conditions. Thus, the final target is to achieve the automatic installation and positional correction of the ribbon bridge during the operation time. A recent study of Yasuhiro and co-authors [2] focused on measurement system for measuring the positional displacement of floating units using a multi-copter that enables the feasibility of automatic installation and self-correction of the floating ribbon bridge by applying control theory. Besides, Mohammad and Yoshiyuki [3] and Shixiao and Weicheng [4] analysed the dynamics of floating bridges. However, the needs of a sufficient mathematical study in order to establish the control-based modelling were not satisfied. In this study, we propose an efficient mathematical model based on analytical modelling of floating units, and identify the system parameters. To verify the feasibility of the abovementioned mathematical modelling and etc., a numerical investigation is studied by applying LQR control technique employing computational software MATLAB and Simulink. The numerical results demonstrate that our proposed model and control strategy have potential ability to enable the automated installation and operation of a new ribbon bridge.

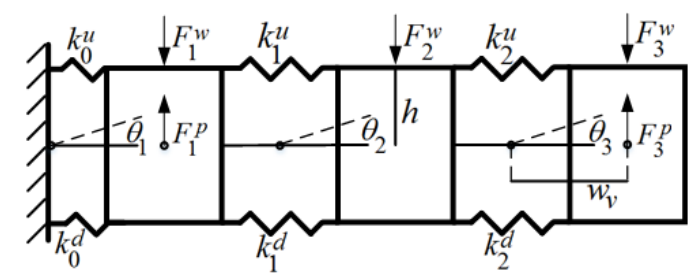

Fig. 1. The structure diagram of Ribbon Bridge.

\section{Problem statement}

\subsection{Mathematical modelling}

The modelling process to obtain a model of the floating units is introduced by authors [5]. Then a basic model consists of the kinematic model and kinetic model [5].

$$
\mathrm{J} \dot{v}+\mathrm{C}_{\mathrm{v}} v=\tau
$$

Where $\boldsymbol{v}=[u, v, r]^{T} \in R^{3}$ describes the surge, sway, and yaw rate of the floating unit motion in body fixed frame; $\mathrm{J} \in R^{3 X 3}$ presents a mass/inertia matrix; $\mathrm{C}_{v}$ is the hydrodynamic damping matrix:

$$
\mathrm{J}=\left[\begin{array}{ccc}
m-X_{u} & 0 & 0 \\
0 & m-Y_{\dot{v}} & 0 \\
0 & -N_{\dot{v}} & I_{z}-N_{\dot{r}}
\end{array}\right] \quad \mathrm{C}_{\mathrm{v}}=\left[\begin{array}{ccc}
-X_{u} & 0 & 0 \\
0 & -Y_{v} & 0 \\
0 & 0 & -N_{r}
\end{array}\right]
$$

Then, the mathematic model for the three-bay ribbon bridge is written as:

$$
\begin{aligned}
& J_{1} \ddot{\theta}_{1}+C_{v} \dot{\theta}_{1}+C_{0} \dot{\theta}_{1}+C_{1}\left(\dot{\theta}_{1}-\dot{\theta}_{2}\right)=\left(F_{0}^{l u}+F_{0}^{l d}+F_{1}^{r u}+F_{1}^{r d}\right) h+\left(F_{1}^{p}-F_{1}^{w}\right) w_{v} \\
& J_{2} \ddot{\theta}_{2}+C_{v} \dot{\theta}_{2}+C_{1}\left(\dot{\theta}_{2}-\dot{\theta}_{1}\right)+C_{2}\left(\dot{\theta}_{2}-\dot{\theta}_{3}\right)=\left(F_{1}^{l u}+F_{1}^{l d}+F_{2}^{r u}+F_{2}^{r d}\right) h+\left(-F_{2}^{w}\right) w_{v} \\
& J_{3} \ddot{\theta}_{3}+C_{v} \dot{\theta}_{3}+C_{2}\left(\dot{\theta}_{3}-\dot{\theta}_{2}\right)+C_{3} \dot{\theta}_{3}=\left(F_{2}^{l u}+F_{2}^{l d}\right) h+\left(F_{3}^{p}-F_{3}^{w}\right) w_{v}
\end{aligned}
$$


Where, $C_{1}, C_{2}, C_{3}$ are damping coefficient of the corresponding joints (in this case, we consider $C_{1}=C_{2}=C_{3}=C$ ); $\theta$ is the yaw rate; $F_{i}^{l u}, F_{i}^{l d}, F_{i}^{r u}, F_{i}^{r d}$ are spring forces of upside and downside in left hand side and right hand side, $h$ is the arm of spring forces; $F_{i}^{p}, F_{i}^{w}$ are forces generated by actuators and the current flow force, and $w_{v}$ is the arm of force generated by driving DC motor and current flow.

Table 1. Specifications of experiment apparatus.

\begin{tabular}{|c|c|c|}
\hline No. & Item & Specification \\
\hline 1 & Load cell & $10 \mathrm{~N}(\max )-5 \mathrm{VDC}$ \\
\hline 2 & Incremental Encoder & 1024 pulse/rev \\
\hline 3 & Motor Driver & KDC248H $-12 \sim 24 \mathrm{~V}$ \\
\hline 4 & DC Motor & $12 \mathrm{~V} / 17.200 \mathrm{rpm}$, Graupner co. \\
\hline 5 & Propeller & $\emptyset 41 \mathrm{~mm}$, Graupner co. \\
\hline
\end{tabular}

\subsection{Parameter identification}

In this study, the 3-bay ribbon bridge pilot model is used for experiment. The modelling of 3-bay ribbon bridge is carried out with regarding individual yaw motion. The identification for the ribbon bridge includes two different steps by conducting several experiments [6].

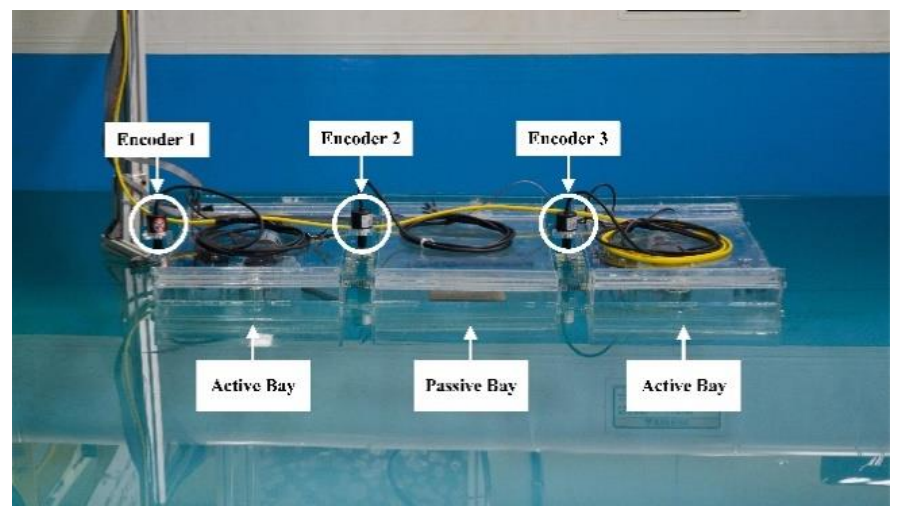

Fig. 2. The ribbon bridge model using for experiment.

The experimental apparatus of ribbon bridge system is constructed as shown in Fig. 2 with 3-bays which are driven by propulsion system with DC motor. Two of three bays have active propulsion system. In Fig. 2, the active bay has a motor driven propulsion system, in other word, there is no propulsion system in the passive bay. The dimensions of each single bay is described by $260 \mathrm{~mm}[\mathrm{~L}] \mathrm{x} 440 \mathrm{~mm}[\mathrm{~W}] \mathrm{x} 80 \mathrm{~mm}[\mathrm{H}]$. The mass of each single bay is $3.79 \mathrm{~kg}$. As mentioned previously, two active bays are driven by propellers which are controlled by the DC motor respectively. In addition, a camera is employed to measure the position and a load cell is used to obtain the driven force generated by the propulsion system. At first, the relation between the force generated by driving DC motor, propeller, and the input voltage is identified by experiments and employing Matlab Identification Toolbox [7]. The obtained transfer function which approximately describes the input-output relation between the input voltage $(\mathrm{V})$ and the generated force $(\mathrm{N})$ is expressed as:

$$
\frac{F_{i}^{p}(s)}{U(s)}=\frac{18.5}{s^{2}+6.915 s+55.74}
$$


Second, the ribbon bay mass matrix and the damping matrix were determined from simulation and collected data by experiment, as shown in Fig. 3. In the results, the parameters are identified as follows:

$$
\begin{aligned}
& J=I_{z}-N_{\dot{r}}=0.0055, \\
& C_{v}=-N_{r}=0.004, \\
& C=0.001
\end{aligned}
$$
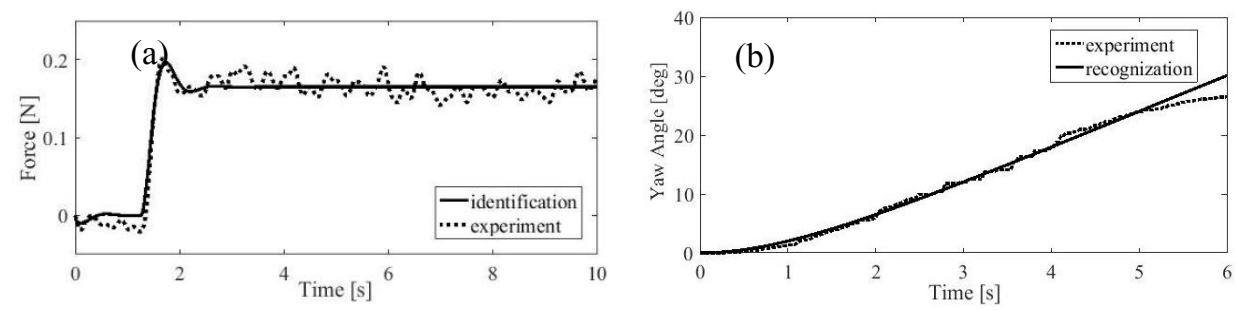

Fig. 3. Comparision studis for system identification by simulation and experiment results. (a)forces produced by propulsion system, (b)free yaw motion responses.

\section{Controller design}

The 3-bay ribbon bridge with two active bays and one passive driven bay can be modelled in the state-space model with respect to following state variables.

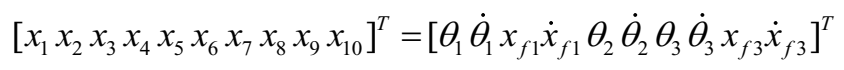

It can be seen that the yaw angles depend on the input driven forces made from the propulsion system. The linear quadratic regulator (LQR) control [8] is a well known design technique which provides marvellous control performance and reduce the effects of disturbance as well as maintaining the robustness of system stability. In this study, the control objective is minimizing the positional displacement. For this, the LQR controller with integral action is designed based on the mathematical modelling of three-bay ribbon bridge system. Let consider the time invariant system:

$$
\begin{aligned}
& \dot{x}(t)=A x(t)+B u(t) \\
& y(t)=C x(t)
\end{aligned}
$$

where $x \in R^{n}$ is the state vector of the system, $u \in R^{m}$ control inputs, $y \in R^{m}$ is output to be regulated, and $A, B$, and $C$ represent the real constant matrices of system. Consider the problem to regulate the output $y(t)$ to the desired pre-set tracking $r(t)$ under the action of the input disturbance $w_{d}(t)$. To obtain good tracking performance, the authors design a servosystem shown in Fig. 4 where an integrator is introduced to reject steady state error.

Then, the control system shown in Fig. 4 can be expressed as:

$$
\begin{aligned}
& {\left[\begin{array}{c}
\dot{x} \\
\dot{w}_{e}
\end{array}\right]=\left[\begin{array}{cc}
A-B K_{f} & B K_{w} \\
-C & 0
\end{array}\right]\left[\begin{array}{c}
x \\
w_{e}
\end{array}\right]+\left[\begin{array}{l}
0 \\
1
\end{array}\right] r} \\
& y=\left[\begin{array}{ll}
C & 0
\end{array}\right]\left[\begin{array}{c}
x \\
w_{e}
\end{array}\right]
\end{aligned}
$$




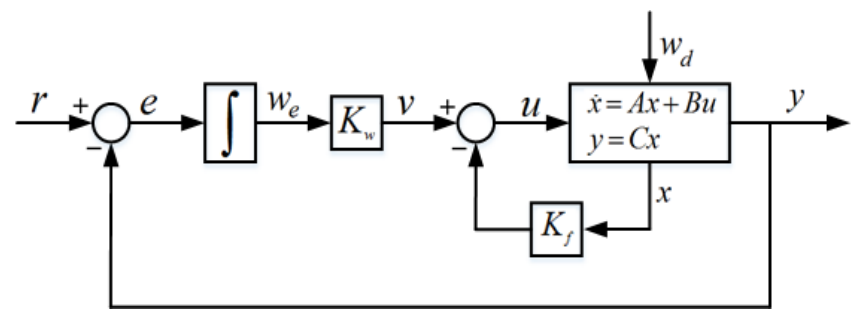

Fig. 4. 3-bay ribbon bridge control system using $L Q R$ controller with integral action.

Our goal is to design an optimal tracking controller that tracks the desired angles of each ribbon bay. In this case, the target is maintaining the linearity of the ribbon bridge by minimizing the positional displacement of each bay. That means the error $e=r(t)-C x(t)$ must approach zero. Following the general optimal control theory, the weighting matrices were chosen as:

$$
\begin{aligned}
& R=\operatorname{diag}\{1.500001 .5010001011\} ; \\
& Q=\operatorname{diag}\{4501.54551 .5\}
\end{aligned}
$$

In the results, an obtained linear control law is given as follows:

$$
u=-K_{f w} \tilde{x}
$$

where,

$$
K_{f w}=-R^{-1} B_{0}{ }^{T} P=\left[\begin{array}{ll}
K_{f} & K_{w}
\end{array}\right]
$$

And, $P$ is a positive definite symmetric matrix that satisfies the following Riccati equation:

$$
P A_{0}+A_{0}^{T} P-P B_{0} R^{-1} B_{0}^{T} P+Q=0
$$

Therefore, $K_{f}$ and $K_{w}$ are:

$K_{f}=\left[\begin{array}{llllllllll}0.0318 & 0.0127 & 0.0432 & 0.0061 & 0.0191 & 0.0135 & 0.0033 & 0.0102 & 0.0349 & 0.0049 \\ 0.0054 & 0.0101 & 0.0345 & 0.0048 & 0.0257 & 0.0223 & 0.0433 & 0.0311 & 0.1063 & 0.0150\end{array}\right]$ $K_{w}=\left[\begin{array}{ccc}0.0136 & 0.0058 & -0.0020 \\ -0.0007 & 0.0063 & 0.0134\end{array}\right]$.

\section{Simulation results}

At first, in this section, the authors present and discuss the numerical simulation results.

The simulation results are shown in Fig. 5. It is the initial response. In Fig. 5, (a) is yaw motion control responses in which the dotted line is the reference signal and others are yaw motions of three bay, respectively. And (b) represents the control inputs which are supplied to the two propulsion systems. Where, wave disturbance attacks the floating units at $5[\mathrm{~s}]$ and $15[\mathrm{~s}]$ with $3[\mathrm{~cm}]$ height. 
The simulation results show that the yaw deviations are quickly approaching to the target point in the presence of disturbance. It confirms that the ability of the proposed controller to maintain the linearity of the bridge system.

From these results, the proposed ribbon bridge control system works well such that the effectiveness of this strategy is verified. This result will be extended to the future study including experiment.

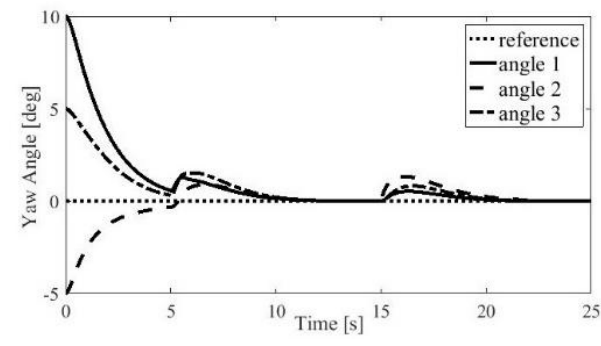

(a) yaw angle responses with disturbance

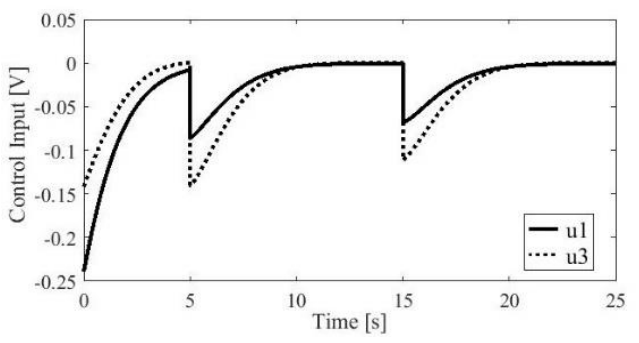

(b) control inputs to the propulsion systems

Fig. 5. Yaw motion control responses and control inputs.

\section{Conclusions}

In this study, the authors have proposed a control strategy for installating the floating bridge system constructed by connecting multi floating units. For the control system design, a servosystem with an integrator is introduced to maintain the linearity of the bridge system and reject the steady state error. The mathematical model for a ribbon bridge system was established by considering the dynamic characteristics and identifying process. In addition, the LQR control approach was developed for the ribbon bridge motion control.

The numerical simulation results demonstrate the effectiveness of the proposed approaching. And it shows that the promising feasibility of positional linearity control, automated installation and operation of floating bridge system without any human power are possible.

This work was supported by the National Research Foundation of Korea (NRF) grant funded by the Korea Government (Ministry of Education) (No.NRF-2015R1D1A1A09056885).

\section{References}

1. Improve Ribbon Bridge, GENERAL DYNAMICS 2-3 (2012)

2. Y. Hirono, Y. Mizuchi, Y-B. Kim, Y-W. Choi, LNEE 135, 18-27 (2016)

3. M. S. Seif, Y. Inoue, Marine Structures 11 (1998)

4. S. Fu, W. Cui, Marine Structures 29, 246-256 (2012)

5. T. I. Fossen, Marine Control System (Trondheim, Norway, 2002)

6. R. Skjetne, O. N. Smogeli, T. I. Fossen, Mod. Ident. Con. 23-1, 3-27 (2004)

7. Matlab System Identification Toolbox User's Guide (The MathWorks, 2016)

8. C. Hajiyev, H. E. Soken, S. Y. Vural, Linear Quadratic Regulator Controller Design, (Springer, 2015) 